\title{
Augmented Reality Visualization
}

with Use of Image Overlay

Technology for MR Imaging-

guided Interventions: Assessment

of Performance in Cadaveric Shoulder and Hip Arthrography at $1.5 \mathrm{~T}^{1}$

Jan Fritz, MD

Paweena U-Thainual, MS

Tamas Ungi, MD, PhD

Aaron J. Flammang, MBA, BSRT (MR)

Gabor Fichtinger, PhD

Iulian I. Iordachita, PhD

John A. Carrino, MD, MPH
Purpose:

Materials and Methods:

Results:

1'From the Russell H. Morgan Department of Radiology and Radiological Science, Johns Hopkins University School of Medicine, 601 N Caroline St, JHOC 5165, Baltimore, MD 21287 (J.F., J.A.C.); Department of Mechanical Engineering and Laboratory for Computational Sensing and Robotics, Johns Hopkins University, Baltimore, Md (P.U.T., I.I.I.); School of Computing, Queen's University, Kingston, Ontario, Canada (P.U.T., T.U., G.F.); and Center for Applied Medical Imaging, Siemens Corporate Research, Baltimore, MD (A.J.F.). Received December 15, 2011; revision requested February 20, 2012; revision received March 16; accepted March 29; final version accepted April 13. Address correspondence to J.A.C. (e-mail: jcarrin2@jhmi.edu).
To prospectively assess overlay technology in providing accurate and efficient targeting for magnetic resonance (MR) imaging-guided shoulder and hip joint arthrography.

A prototype augmented reality image overlay system was used in conjunction with a clinical 1.5-T MR imager. A total of 24 shoulder joint and 24 hip joint injections were planned in 12 human cadavers. Two operators (A and B) participated, each performing procedures on different cadavers using image overlay guidance. MR imaging was used to confirm needle positions, monitor injections, and perform MR arthrography. Accuracy was assessed according to the rate of needle adjustment, target error, and whether the injection was intraarticular. Efficiency was assessed according to arthrography procedural time. Operator differences were assessed with comparison of accuracy and procedure times between the operators. Mann-Whitney $U$ test and Fisher exact test were used to assess group differences.

Forty-five arthrography procedures (23 shoulders, 22 hips) were performed. Three joints had prostheses and were excluded. Operator A performed 12 shoulder and 12 hip injections. Operator B performed 11 shoulder and 10 hip injections. Needle adjustment rate was $13 \%$ (six of 45; one for operator $\mathrm{A}$ and five for operator B). Target error was $3.1 \mathrm{~mm} \pm 1.2$ (standard deviation) (operator A, $2.9 \mathrm{~mm} \pm 1.4$; operator B, $3.5 \mathrm{~mm} \pm 0.9)$. Intraarticular injection rate was $100 \%$ (45 of 45 ). The average arthrography time was 14 minutes (range, 6-27 minutes; 12 minutes [range, 6-25 minutes] for operator A and 16 minutes [range, 6-27 min] for operator B). Operator differences were not significant with regard to needle adjustment rate $(P=.08)$, target error $(P=.07)$, intraarticular injection rate $(P>.99)$, and arthrography time $(P=.22)$.

Conclusion: Image overlay technology provides accurate and efficient MR guidance for successful shoulder and hip arthrography in human cadavers.

${ }^{\circ}$ RSNA, 2012

Supplemental material: http://radiology.rsna.org/lookup /suppl/doi:10.1148/radiol.12112640/-/DC1 
D rect magnetic resonance (MR) arthrography requires the coordination of joint injection and diagnostic MR imaging, which are typically not colocated (1). Furthermore, fluoroscopy or computed tomographic (CT) guidance $(2,3)$ result in exposure to ionizing radiation and its associated health risks (4-6). Interventional MR imaging can overcome these limitations by combining joint injection and MR imaging in a single setting (7-9); however, this typically requires targeting and puncture inside the bore of the magnet $(1,7,10)$. Augmented reality (AR) navigation technology instead can provide MR imaging-guided targeting and joint puncture outside the magnet bore.

The purpose of this study was to demonstrate that AR image overlay technology can provide accurate, efficient, and reliable targeting for MR-guided shoulder and hip joint arthrography.

\section{Materials and Methods}

\section{System Description}

A prototype MR-compatible, two-dimensional, AR image overlay system was used in conjunction with a clinical 1.5-T MR imaging system (Magnetom Espree; Siemens Healthcare, Erlangen, Germany) (Fig 1a) (10). The virtual needle paths were planned on the MR images of the target joint by using the PerkStation employing 3D Slicer software (version 3.6; http://www.slicer.org) (11) (Figs 1b, 2a). The respective MR image with the virtual needle path was then projected onto the subject at the appropriate location. The operator then punctured the joint by maneuvering the needle along the virtual needle path (Fig 1c, Movie 1 [online]).

\section{Subjects}

The shoulder and hip joints of 12 nonembalmed, whole-spine torso human

\section{Advance in Knowledge}

- Augmented reality image overlay is a reality technology that may facilitate the targeting of MRguided shoulder and hip joint injections. cadavers (seven women, five men; age range at death, 50-99 years; mean age at death, 75 years) were used. Four of $12(33 \%)$ cadavers were smaller size (living body mass index [BMI], 16-18.5 $\mathrm{kg} / \mathrm{m}^{2}$ ), four $(33 \%)$ were medium size (18.5-25 kg/m² BMI), and four (33\%) were larger size (25-30 kg/m² BMI) (12). All cadavers were obtained and used in accordance with institutional and Health Insurance Portability and Accountability Act guidelines. The frozen cadaveric subjects were allowed to thaw for 24 hours at room temperature (approximately $20^{\circ}-22^{\circ} \mathrm{C}$ ) prior to MR imaging.

\section{Joint Injections}

A total of 24 shoulder joint injections and 24 hip joint injections were planned. In each subject, two shoulder joint injections and two hip joint injections were attempted. Each joint was injected once. The study was performed on 12 different days (one subject per day) during a period of 14 weeks. Joint injections were performed by two operators (operator A, J.F., with 10 years and operator B, J.A.C., with 15 years of experience in interventional MR and arthrography procedures). To become familiar with the system and the workflow, specific training was accomplished by each operator prior to the experiment by using the image overlay system on 10 gel phantom targets. All cadaver targets were prospectively, nonselectively, and evenly assigned. Shoulder joints were targeted through the rotator interval by using an anterior or anterolateral approach $(2,3)$. Hip joints were targeted at the femoral head-neck junction with a modified lateral approach by using an anterolateral needle path (13).

\section{Implications for Patient Gare}

- With image overlay technology, joint injections and diagnostic MR imaging can be combined into a single procedure.

- With image overlay technology, patient and operator exposure to ionizing radiation can be obviated.

\section{Workflow}

Subjects were placed supine on the table of the MR imaging system. In addition to the table coil elements, a flexible loop-shaped radiofrequency surface coil (Siemens Healthcare) with a diameter of $19 \mathrm{~cm}$ was placed over the target site. Three-dimensional sampling perfection with application optimized contrast using different flip angle evolutions (SPACE) sequences was used for planning shoulder (repetition time msec/echo time msec, 1000/ 34; voxel size, $1 \times 1$ $\times 1 \mathrm{~mm}$; acquisition time, 2 minutes 55 seconds) and hip joint (1100/100; voxel size, $1 \times 1 \times 1 \mathrm{~mm}$; acquisition time, 5 minutes 43 seconds) injections, respectively.

The isotropic three-dimensional MR imaging data were subsequently imported into the 3D Slicer software. The image overlay system was then calibrated for the operator by aligning the overlay projection with the operator's line of sight by using a MR-compatible, in-room keyboard in conjunction with the PerkStation module of 3D Slicer software. Next, the operator evaluated the isotropic MR images at the image

\section{Published online before print \\ 10.1148/radiol.12112640 Content codes: MR MK \\ Radiology 2012; 265:254-259 \\ Abbreviations: \\ $\mathrm{AR}=$ augmented reality \\ $\mathrm{Cl}=$ confidence interval \\ SPACE = sampling perfection with application optimized contrast using different flip angle evolutions}

\section{Author contributions:}

Guarantors of integrity of entire study, J.F., A.J.F., J.A.C.; study concepts/study design or data acquisition or data analysis/interpretation, all authors; manuscript drafting or manuscript revision for important intellectual content, all authors; approval of final version of submitted manuscript, all authors; literature research, J.F., T.U., A.J.F.; clinical studies, T.U., A.J.F., I.I.I.; experimental studies, J.F., T.U., A.J.F., I.I.I., J.A.C.; statistical analysis, J.F., T.U., A.J.F.; and manuscript editing, J.F., T.U., A.J.F., G.F., I.I.I., J.A.C.

\section{Funding:}

This research was supported by the National Cancer Institute (grant 1 R01 CA118371-01A2).

Potential conflicts of interest are listed at the end of this article. 


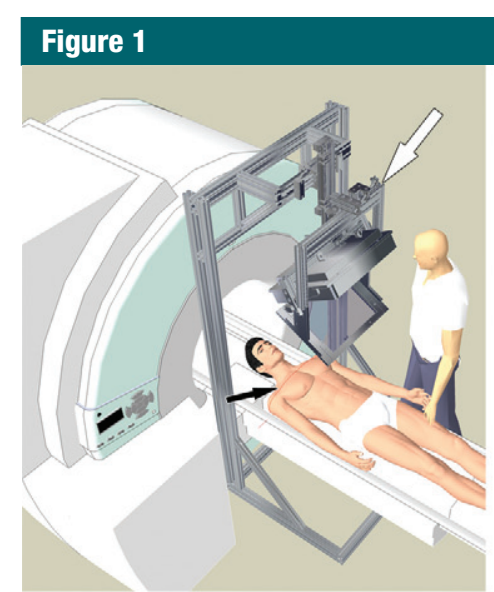

a.
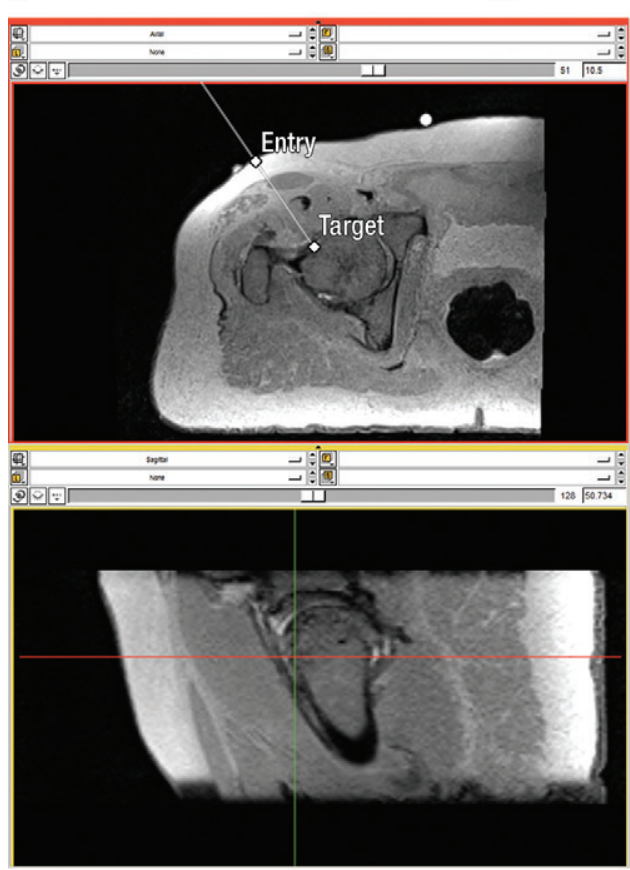

b.

Figure 1: (a) Schematic depiction of the interventional setup of the AR image overlay prototype system (white arrow) in conjunction with a clinical 1.5-T MR imaging system. The red laser line (black arrow) on the subject's skin coincides with the selected MR image target. (b) Planning of the needle path to the femoral head-neck junction with three-dimensional SPACE MR imaging (1100/100; flip angle, $120^{\circ}$; two signals acquired; echo train length, 117 ; voxel size, $1 \times 1 \times 1 \mathrm{~mm}$; number of sections, 60; field of view, $256 \times 224$ mm; base resolution, 192 pixels; phase resolution, 100\%; bandwidth, $751 \mathrm{~Hz}$; acquisition time, 5 minutes 43 seconds) data set. Top left: Axial reformation. Top right: Three-dimensional map. Bottom left: Sagittal reformation. Bottom right: Coronal reformation. $A=$ anterior, $R=$ right, $L=$ left, $S=$ superior, $I=$ inferior. (c) Intraprocedural photograph of MR-guided injection of the right hip with image overlay system, from the operator's view. The target MR image (white arrow) is fused with a graphical representation of the planned needle path and depth (gray arrow). The skin entry point is indicated by the apparent intersection of the red laser line and the virtual needle path. Black arrow = operator's hand while inserting the needle (Movie 1 [online]).

overlay system workstation and defined the anatomic target site and the skin entry point (Fig 2a). The PerkStation software module calculated and displayed the virtual needle path and the insertion depth.
Needle placement was performed with AR MR guidance and simultaneous visualization of the joint and the acquired MR image (including the virtual needle path) by looking through the semitransparent mirror. The operator identified the surface entry point according to the intersection of the image overlay system laser and the displayed virtual needle path. MR-compatible, 5- or 10-cm, 22-gauge Lufkin needles (EZ-Em, Lake Success, NY) were used. An individually adjustable clip-on depth gauge was used to label anticipated insertion depths.

After the needle was placed, axial intermediate-weighted turbo spinecho MR images (1200/12; acquisition time, 22 seconds) were obtained of the joint for visual assessment of the needle tip locations (Fig 2b). After evaluation of the images by the operator, needle adjustments were performed, if necessary, followed by acquisition of additional intermediate-weighted turbo spin-echo images.

Once the needle was deemed to be in satisfactory location by the operator, a 20 -cm extension tube with a syringe was connected. The table was moved into the isocenter of the magnet bore for injection of $10 \mathrm{~mL}$ of gadolinium-enhanced saline solution. The injection was monitored with real-time MR imaging by using a continuously acquired and displayed single-section T1-weighted fast low-angle shot two-dimensional MR imaging sequence (9.3/3.5; acquisition time, 1 second) (Movie 2 [online]). The operator monitored the injection process on a monitor of an in-room console. Finally, a T1-weighted turbo spin-echo sequence (500/12; acquisition time, 2 minutes 5 seconds) with chemical fat saturation was performed of the respective joint (Fig 2c).

\section{Assessment of Technical Performance Parameters}

We assessed the following technical performance parameters: needle adjustment rate, target error, intraarticular injection rate, and arthrography time.

Needle adjustments included straight needle advancement, change of needle 
trajectory, and removal and new placement after the initial placement.

The target error was defined as the Euclidean distance between the planned and the final position of the needle tip. The PerkStation was used for calculations by comparing the planned needle tip location with the true needle tip location as manually determined by the needle artifact on final proton densityweighted turbo spin-echo MR images (11). Measurements were performed three times by a single individual (J.F.). Intrarater variability was assessed by using coefficient of variation $(\mathrm{CV})$ as follows: $\mathrm{CV}=\sigma / \mu$, where $\sigma$ is the $1 \mathrm{st}$ standard deviation and $\mu$ is the arithmetic mean.

Successful intraarticular injection was defined as the complete accumulation of the injectant inside the joint capsule. Two board-certified radiologists (H.B. and A.K.R.) independently categorized the contrast agent distributions into completely intraarticular, incompletely intraarticular, or extraarticular distribution using a picture archiving and communication system workstation. Minimal reflux along the needle track was considered acceptable for a completely intraarticular joint injection.

The length of time for planning of the needle path (planning time), joint puncture (puncture time), MR imaging control of the needle position (control time), and injection of the contrast agent (injection time) were recorded as the task times. Arthrography time was defined as the sum of the planning, insertion, control, and injection times.

\section{Statistical and Quantitative Assessments}

Accuracy was assessed according to needle adjustment rate, target error, and intraarticular injection rate. Efficiency was assessed according to the arthrographic procedure time. Systematic differences between the operators were assessed by comparing accuracy and procedure times. Statistical analysis was performed with a statistical software package (JMP, version 7.01; SAS Institute, Cary, NC). Quantitative variables were either expressed
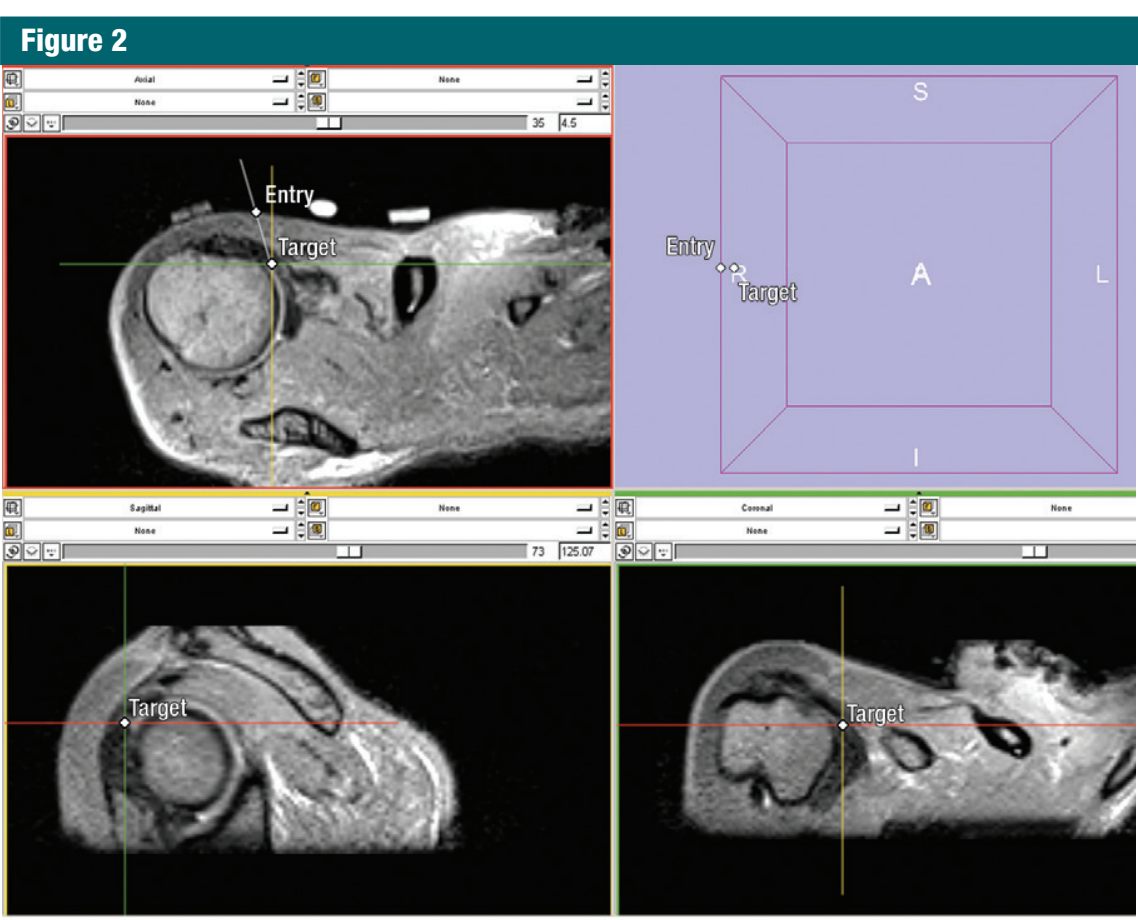

a.
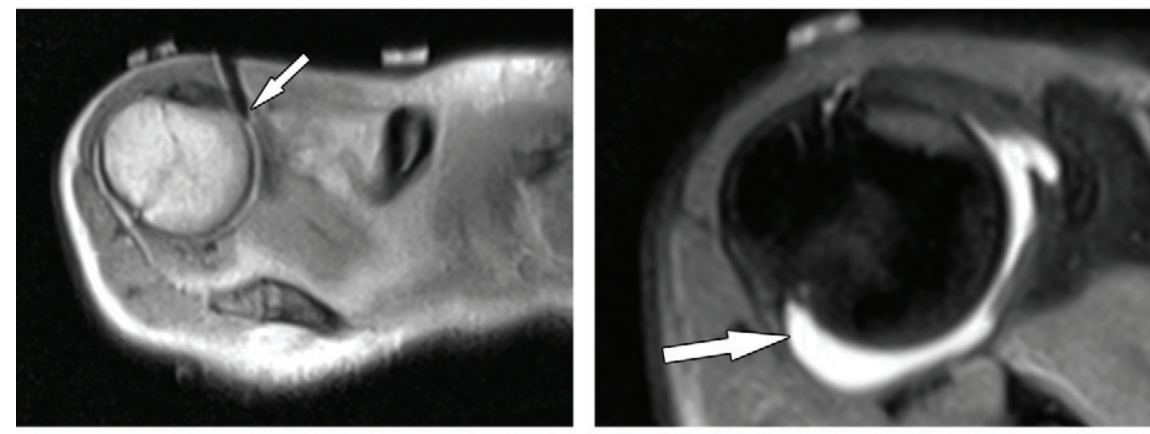

b.

c.

Figure 2: Image overlay MR-guided injection and arthrography of the right shoulder. (a) Planning of the needle path through the rotator interval with three-dimensional SPACE MR imaging (1000/34; flip angle, $120^{\circ}$; two signals acquired; echo train length, 49 ; voxel size, $1 \times 1 \times 1 \mathrm{~mm}$; number of sections, 60 ; field of view, $192 \times 168 \mathrm{~mm}$; base resolution, 192 pixesl; phase resolution, 100\%; bandwidth, $744 \mathrm{~Hz}$; acquisition time, 2 minutes 55 seconds) data set. Top left: Axial reformation. Top right: Three-dimensional map. Bottom left: Sagittal reformation. Bottom right: Coronal reformation. $A=$ anterior, $R=$ right, $L=$ left, $S=$ superior, $I$ = inferior. (b) Axial intermediate-weighted turbo spin-echo MR image shows the needle tip (arrow) at the target location. (c) Axial T1-weighted diagnostic MR arthrogram with intraarticular contrast agent (arrow) following joint injection (Movie 2 [online]).

as arithmetic mean \pm 1 standard deviation or as the median (14). Minimum and maximum values were given in parenthesis. The assessment of operator differences of the target error was based on individual assessments of the target errors. All of the duplicated assessments were included in the summary statistics of the target error. Categorical variables were expressed as frequencies and proportions. The Mann-Whitney $U$ test and Fisher exact test were used to compute group differences. Confidence intervals (CIs) 


\begin{tabular}{|c|c|c|c|c|c|}
\hline \multicolumn{6}{|c|}{ Arthrography Times according to Task } \\
\hline Task & Average Time & Shoulder & Hip & Operator A & Operator B \\
\hline Planning (min) & $2(1-5)$ & $2(1-5)$ & $2(1-5)$ & $2(1-4)$ & $3(1-5)$ \\
\hline Puncture (min) & $5(1-9)$ & $4(1-7)$ & $5(1-9)$ & $3.5(1-9)$ & $5(1-9)$ \\
\hline Control (min) & $2(1-6)$ & $2(1-6)$ & $2(1-5)$ & $2(1-6)$ & $2(1-5)$ \\
\hline Injection (min) & $5(2-11)$ & $4(2-11)$ & $5(3-10)$ & $4.5(2-11)$ & $5(2-10)$ \\
\hline Arthrography time $(\mathrm{min})^{*}$ & $14(6-27)$ & $11(6-25)$ & $15.5(7-27)$ & $12(6-25)$ & $16(6-27)$ \\
\hline
\end{tabular}

are provided for the mean differences between the operators. A $P$ value of less than .05 was considered to indicate a significant difference. A post hoc power analysis of the arthrography time was performed to calculate the probability of detecting a significant difference between the operators.

\section{Results}

A total of 45 joint injection procedures were performed, of which 23 (51\%) were shoulder injections (operator A, 12 of $23,52 \%$; operator B, 11 of 23 , $48 \%)$ and 22 (49\%) were hip joint injections (operator A, 12 of 22, 54\%; operator B, 10 of 22, 46\%). Three subjects had joint prostheses, which resulted in distorted MR image and prevented MR image guidance. Six (13\%) needle adjustments (operator A, $n=1$; operator $\mathrm{B}, n=5$ ) were performed in six different joints. The needle adjustment rate was not significantly different between the operators $(P=.08)$.

Completely intraarticular injections were achieved in all 45 targeted joints $(100 \%, P>.99)$. There were no interobserver assessment differences. The target error was $3.1 \mathrm{~mm} \pm 1.2$ (range, 0.9-6.4 mm; coefficient of variation, $11.5 \% \pm 4.8$; range, $2.7 \%-22.7 \%$ ). The target error for operator A was 2.9 $\mathrm{mm} \pm 1.4$ (range, $0.9-6.4 \mathrm{~mm}$ ). The target error for operator B was $3.5 \mathrm{~mm}$ \pm 0.9 (range, $1.7-5.0 \mathrm{~mm}$ ). The target error was not significantly different between the operators $(P=.07$; difference, -0.62 ; 95\% CI: $-1.33,0.08)$ and targets $(P=.74$; difference, $0.03 ; 95 \%$
CI: $-0.72,0.78)$. The average arthrography time was 14 minutes (range, 6-27 minutes) (Table). There was no significant difference between the operators $(P=.22$; difference, $-2.28 ; 95 \%$ CI: $-5.64,1.08)$. The post hoc power was 0.65 .

\section{Discussion}

Our investigation demonstrated that AR image overlay technology provides accurate MR imaging guidance for intraarticular injection of contrast material into shoulder and hip joints. Image overlay technology combines joint injection and MR imaging into a single procedure, thereby avoiding a more complex scheduling process, possible exposure to ionizing radiation, and the need for an additional colocated modality. The availability of image overlay navigation allows performance of procedures at outpatient imaging centers without access to a fluoroscopy unit and can obviate CT guidance for joint injections (2).

The following time requirements have been reported for injection: 5-8 minutes with fluoroscopic guidance (2), and approximately 5 minutes (average) with sonographic guidance (16). These reported times lack detailed description, and it is not certain if all arthrography-related tasks were included in the measurements. By taking into consideration the time-saving effects of colocating the joint injection and MR imaging, the length of time needed for arthrography (14 minutes) would be competitive with the alternatives. (15), 2-26 minutes with CT guidance
Performing MR-guided joint injections with AR navigation obviates the requirement of a dedicated interventional MR imaging system and overcomes the limited access to the puncture site inside the magnet bore (1,7,17-19). Image overlay technology realizes simultaneous visualization of the MR images and the target. The combination of MR image and target results in a "look and feel" environment similar to a surgical procedure and an intuitive hand-eye coordination, which can simplify imageguided puncture. This is in distinction to the spatial and visual separation of the MR image display and targeting (20-24). The concept of AR image overlay navigation could be used in conjunction with other interventional MR imaging procedures, such as softtissue and osseous biopsy, as well as injection procedure of the spine and pelvis. Additional studies are required to confirm this hypothesis.

The target error of approximately 3 $\mathrm{mm}$ provided sufficient clinical usefulness for needle placement and intraarticular injections. The accuracy of the image overlay system appears within the range of that of other systems, which were reported between 1.1-7.5 mm (20-25), allowing for differences in error assessments. Although our results are encouraging, our study had limitations. Because of the use of cadavers, patient motion and respiration were not present. Although shoulder and hip injections are usually less affected by respiration, the influence on technical performance of a static AR system such as the image overlay is currently unknown. Operator differences are at least partially confounded by differences between cadavers that were compared, and it may be impossible to discern whether this confounding led to an under- or overassessment. The power of target error was $65 \%$, suggesting that our study was underpowered to detect nonsignificant operator differences. We used experienced operators who were familiar with the image overlay system and trained in the use of the system prior to the experiments, which may have influenced the average arthrography time. 
On the basis of the results of this study, we plan to extend our work to assess technical efficacy in an upcoming clinical trial. This AR technique has the potential to simplify the workflow of direct MR arthrography in patients by combining joint injection and diagnostic high-field-strength MR imaging into a single procedure, while avoiding exposure to ionizing radiation of patients and operators.

Acknowledgments: The authors thank Harald Brodoefel, MD, and Ajay K. Ravi, MD, for their evaluations.

Disclosures of Potential Conflicts of Interest: J.F. No potential conflicts of interest to disclose. P.U.T. Financial activities related to the present article: author received money for travel from Natural Sciences and Engineering Research Council of Canada. Financial activities not related to the present article: none to disclose. Other relationships: none to disclose. T.U. No potential conflicts of interest to disclose. A.J.F. Financial activities related to the present article: none to disclose. Financial activities not related to the present article: author has a portion of his retirement funding in Siemens stock. Other relationships: none to disclose. G.F. Financial activities related to the present article: institution has a research subcontract from NIH (NIH RO1CA118371-04). Financial activities not related to the present article: none to disclose. Other relationships: none to disclose. I.I.I. Financial activities related to the present article: none to disclose. Financial activities not related to the present article: institution received grant from NIH, NSF; institution has patent from Sentinelle Medical, Toronto(technology transfer for patent U.S. Provisional Application Serial No. 60/782,705 filed March 14, 2006, Publication No. WO/2007/106558, 09/20/2007; institution receives royalties from Gulmay Medical; institution received funding for research in cochlear electrode insertion from Cochlear Corporation. Other relationships: none to disclose. J.A.C. Financial activities related to the present article: none to disclose. Financial activities not related to the present article: author is a board member of Vital, consultant to Quality Medical Metics and Medtronic, received money for multiple medical-legal activities; institution received grants from Siemens, Toshiba, Carestream, and Integra; author received payment for multiple grand rounds and invited lectures with honoraria; author has stock in Merge Healthcare. Other relationships: none to disclose.

\section{References}

1. Miller TT. MR arthrography of the shoulder and hip after fluoroscopic landmarking. Skeletal Radiol 2000;29(2):81-84.
2. Mulligan ME. CT-guided shoulder arthrography at the rotator cuff interval. AJR Am J Roentgenol 2008;191(2):W58-W61.

3. Dépelteau H, Bureau NJ, Cardinal E, Aubin B, Brassard P. Arthrography of the shoulder: a simple fluoroscopically guided approach for targeting the rotator cuff interval. AJR Am J Roentgenol 2004;182(2):329-332.

4. Steinbach LS, Palmer WE, Schweitzer ME. Special focus session: MR arthrography. RadioGraphics 2002;22(5):1223-1246.

5. Nawfel RD, Judy PF, Silverman SG, Hooton S, Tuncali K, Adams DF. Patient and personnel exposure during CT fluoroscopyguided interventional procedures. Radiology 2000;216(1):180-184.

6. Paulson EK, Sheafor DH, Enterline DS, McAdams HP, Yoshizumi TT. CT fluoroscopyguided interventional procedures: techniques and radiation dose to radiologists. Radiology 2001;220(1):161-167.

7. Petersilge CA, Lewin JS, Duerk J, Hatem SF. MR arthrography of the shoulder: rethinking traditional imaging procedures to meet the technical requirements of MR imaging guidance. AJR Am J Roentgenol $1997 ; 169(5): 1453-1457$.

8. Graves MJ, Wakely S, Bearcroft PW, et al. MR-guided direct arthrography of the hip. J Magn Reson Imaging 2008;28(2): 462-465.

9. Soh E, Bearcroft PW, Graves MJ, Black R, Lomas DJ. MR-guided direct arthrography of the glenohumeral joint. Clin Radiol 2008; 63(12):1336-1341; discussion 1342-1343

10. Fritz J, U-Thainual P, Ungi T, et al. Augmented reality visualization with image overlay for MRI-guided intervention: accuracy for lumbar spinal procedures with a 1.5-T MRI system. AJR Am J Roentgenol 2012;198(3):W266-W273.

11. Vikal S, U-Thainual P, Carrino JA, Iordachita I, Fischer GS, Fichtinger G. Perk Station: percutaneous surgery training and performance measurement platform. Comput Med Imaging Graph 2010;34(1):19-32.

12. Obesity: preventing and managing the global epidemic. Report of a WHO consultation. World Health Organ Tech Rep Ser 2000;894:i-xii, 1-253.

13. Kilcoyne RF, Kaplan P. The lateral approach for hip arthrography. Skeletal Radiol 1992;21(4):239-240.
14. Sonnad SS. Describing data: statisti cal and graphical methods. Radiology 2002;225(3):622-628.

15. Redondo MV, Berná-Serna JD, Campos PA, et al. MR arthrography of the shoulder using an anterior approach: optimal injection site. AJR Am J Roentgenol 2008;191(5): 1397-1400

16. Valls R, Melloni P. Sonographic guidance of needle position for MR arthrography of the shoulder. AJR Am J Roentgenol 1997;169(3):845-847.

17. Trattnig S, Breitenseher M, Rand T, et al. MR imaging-guided MR arthrography of the shoulder: clinical experience on a conventional closed high-field system. AJR Am J Roentgenol 1999;172(6):1572-1574

18. Weiss CR, Nour SG, Lewin JS. MR-guided biopsy: a review of current techniques and applications. J Magn Reson Imaging 2008;27(2):311-325.

19. Fritz J, Thomas C, Clasen S, Claussen CD, Lewin JS, Pereira PL. Freehand realtime MRI-guided lumbar spinal injection procedures at $1.5 \mathrm{~T}$ : feasibility, accuracy, and safety. AJR Am J Roentgenol 2009;192(4):W161-W167.

20. Hata N, Tokuda J, Hurwitz S, Morikawa S MRI-compatible manipulator with remotecenter-of-motion control. J Magn Reson Imaging 2008;27(5):1130-1138.

21. Tokuda J, Fischer GS, Csoma C, et al. Software strategy for robotic transperineal prostate therapy in closed-bore MRI. Med Image Comput Comput Assist Interv 2008;11(Pt 2):701-709.

22. Christoforou E, Akbudak E, Ozcan A, Karanikolas $M$, Tsekos NV. Performance of interventions with manipulator-driven realtime MR guidance: implementation and initial in vitro tests. Magn Reson Imaging 2007;25(1):69-77.

23. Busse H, Garnov N, Thörmer G, et al. Flex ible add-on solution for MR image-guided interventions in a closed-bore scanner en vironment. Magn Reson Med 2010;64(3): 922-928.

24. Wacker FK, Vogt S, Khamene A, et al An augmented reality system for MR image-guided needle biopsy: initial results in a swine model. Radiology 2006;238(2): 497-504.

25. Silverman SG, Collick BD, Figueira MR, et al. Interactive MR-guided biopsy in an openconfiguration MR imaging system. Radiology 1995;197(1):175-181. 